\title{
Conditions for strong ellipticity and M-eigenvalues*
}

\author{
Liqun QI ${ }^{1}$, Hui-Hui DAI ${ }^{2}$, Deren $\mathbf{H A N}^{3}$
}

1 Department of Applied Mathematics, The Hong Kong Polytechnic University, Hong Kong, China

2 Department of Mathematics, The City University of Hong Kong, Hong Kong, China

3 School of Mathematics and Computer Sciences, Nanjing Normal University, Nanjing 210097, China

(C) Higher Education Press and Springer-Verlag 2009

\begin{abstract}
The strong ellipticity condition plays an important role in nonlinear elasticity and in materials. In this paper, we define M-eigenvalues for an elasticity tensor. The strong ellipticity condition holds if and only if the smallest M-eigenvalue of the elasticity tensor is positive. If the strong ellipticity condition holds, then the elasticity tensor is rank-one positive definite. The elasticity tensor is rank-one positive definite if and only if the smallest Z-eigenvalue of the elasticity tensor is positive. A Z-eigenvalue of the elasticity tensor is an M-eigenvalue but not vice versa. If the elasticity tensor is second-order positive definite, then the strong ellipticity condition holds. The converse conclusion is not right. Computational methods for finding M-eigenvalues are presented.
\end{abstract}

Keywords Elasticity tensor, strong ellipticity, M-eigenvalue, Z-eigenvalue MSC 74B99, 15A18, 15A69

\section{Introduction}

The two/three-dimensional field equations for a homogeneous compressible nonlinearly elastic material for static problems without body forces can be written as (see, e.g., Knowles and Sternberg Refs. [8,22])

$$
a_{i j k l}(1+\nabla \boldsymbol{u}) u_{k, l j}=0,
$$

where $u_{i}(\boldsymbol{X})(i=1,2$ or $1,2,3)$ is the displacement field $(\boldsymbol{X}$ is the coordinate of a material point in the reference configuration $), \mathscr{A}=\left(a_{i j k l}\right)$ is the elasticity

* Received January 13, 2009; accepted January 24, 2009

Corresponding author: Liqun QI, E-mail: maqilq@polyu.edu.hk 
tensor, and

$$
a_{i j k l}(\boldsymbol{F})=a_{k l i j}(\boldsymbol{F})=\frac{\partial^{2} W(\boldsymbol{F})}{\partial F_{i j} \partial F_{k l}}, \quad \boldsymbol{F}=1+\nabla \boldsymbol{u},
$$

where $W$ is the strain energy function. We say that the above equations are elliptic at the point $\boldsymbol{X}$ if and only if

$$
\operatorname{det}\left[a_{i j k l}(\boldsymbol{F}(\boldsymbol{X})) y_{j} y_{l}\right] \neq 0
$$

for all unit vectors $y \in \mathbb{R}^{n}, n=2$ or 3 . On the other hand, we say that they are strongly elliptic if and only if

$$
f(x, y) \equiv \mathscr{A} x y x y \equiv \sum_{i, j, k, l=1}^{n} a_{i j k l} x_{i} y_{j} x_{k} y_{l}>0
$$

for all unit vectors $x, y \in \mathbb{R}^{n}, n=2$ or 3 . While for an isotropic material, some inequalities have been established to judge strong ellipticity (see, Refs. $[7,8,22,23,26]$ ), for a general nonlinearly elastic material, a workable criterion to make a judgement is still lacking. Also, it is important to find out along which of the directions $x$ and $y$ the strong ellipticity fails, as this is related to the appearance of solutions with discontinuous strain gradients; see Refs. [7,8]. In this paper, we shall take a different approach from those in the literature mentioned above to tackle the problem of the strong ellipticity and also to give an algorithm for computing the most possible directions along which the strong ellipticity can fail.

Clearly, the strong ellipticity condition holds if and only if the optimal value of the following global polynomial optimization problem is positive:

$$
\begin{array}{ll}
\min & f(x, y) \equiv \mathscr{A} x y x y \equiv \sum_{i, j, k, l=1}^{n} a_{i j k l} x_{i} y_{j} x_{k} y_{l} \\
\text { s.t. } & x^{\mathrm{T}} x=1, \quad y^{\mathrm{T}} y=1,
\end{array}
$$

where $x, y \in \mathbb{R}^{n}, n=2$ or 3 . Because of the form of (4), we may also assume that for any $i, j, k, l$, we have

$$
a_{i j k l}=a_{k j i l}=a_{i l k j}
$$

Hence, $\mathscr{A}$ has 9 independent elements for $n=2$ and 36 independent elements for $n=3$. Denote $\mathscr{A} \cdot y x y$ as a vector whose $i$ th component is

$$
\sum_{j, k, l=1}^{n} a_{i j k l} y_{j} x_{k} y_{l}
$$

and $\mathscr{A} x y x \cdot$ as a vector whose $l$ th component is

$$
\sum_{i, j, k=1}^{n} a_{i j k l} x_{i} y_{j} x_{k}
$$


The optimality condition of (4) is

$$
\left\{\begin{array}{l}
\mathscr{A} \cdot y x y=\lambda x, \\
\mathscr{A} x y x \cdot=\mu y, \\
x^{\mathrm{T}} x=1 \\
y^{\mathrm{T}} y=1 .
\end{array}\right.
$$

Suppose $\lambda, \mu, x$ and $y$ satisfy (5). It is easy to see that

$$
\lambda=\mathscr{A} x y x y=\mu
$$

Thus, we may rewrite (5) as

$$
\left\{\begin{array}{l}
\mathscr{A} \cdot y x y=\lambda x \\
\mathscr{A} x y x \cdot=\lambda y \\
x^{\mathrm{T}} x=1 \\
y^{\mathrm{T}} y=1
\end{array}\right.
$$

If $\lambda \in \mathbb{R}, x, y \in \mathbb{R}^{n}$ satisfy (6), we call $\lambda$ an M-eigenvalue of $\mathscr{A}$, and call $x$ and $y$ left and right M-eigenvectors of $\mathscr{A}$, associated with the M-eigenvalue $\lambda$. Here, the letter ' $M$ ' stands for mechanics.

It is easy to see that M-eigenvalues always exist and the strong ellipticity condition holds if and only if the smallest M-eigenvalue of $\mathscr{A}$ is positive. In Section 2, we study properties of M-eigenvalues.

The strong ellipticity is related to two kinds of positive definiteness of $\mathscr{A}$.

The elasticity tensor $\mathscr{A}$ is called rank-one positive definite if for all $x \in$ $\mathbb{R}^{n}, x \neq 0$,

$$
f(x, x) \equiv \mathscr{A} x^{4} \equiv \mathscr{A} x x x x \equiv \sum_{i, j, k, l=1}^{n} a_{i j k l} x_{i} x_{j} x_{k} x_{l}>0 .
$$

Clearly, if the strong ellipticity holds, then $\mathscr{A}$ is rank-one positive definite. In Section 3, we study the relationships between the strong ellipticity and the rank-one positive definiteness, and their relationships with eigenvalues of $\mathscr{A}$. The elasticity tensor $\mathscr{A}$ is rank-one positive definite if and only if its smallest $\mathrm{Z}$-eigenvalue is positive. The Z-eigenvalues of a tensor were introduced in the fully symmetric case in Ref. [16], extended to a nonsymmetric case in Ref. [18], and further discussed in Refs. [14,17]. In Section 3, we will see that all the Z-eigenvalues of $\mathscr{A}$ are M-eigenvalues of $\mathscr{A}$, but not vice versa. This reveals their relationships.

We may also call $\mathscr{A}$ positive definite if for any $D=\left(d_{i j}\right) \in \mathbb{R}^{n \times n}, D \neq 0$, we have

$$
\mathscr{A} D^{2} \equiv \sum_{i, j, k, l=1}^{n} a_{i j k l} d_{i j} d_{k l}>0 \text {. }
$$


To distinguish it from the rank-one positive definiteness, we call it the secondorder positive definiteness. It is clear that if $\mathscr{A}$ is second-order positive definite, then the strong ellipticity holds as we may regard $d_{i j} \equiv x_{i} y_{j}$ to connect these two concepts. In Section 4, we study the relationship between strong ellipticity and the second-order positive definiteness.

For $n=2$, all the M-eigenvalues of $\mathscr{A}$ and their corresponding left and right M-eigenvectors can be calculated by a direct method. We present such a direct method in Section 5.

For $n=3$, we may find the smallest M-eigenvalue and its corresponding left and right M-eigenvectors by the SOS (sum of squares) method. We describe the SOS method for this problem in Section 6 .

Some final remarks are made in Section 7.

\section{M-Eigenvalues of a fourth-order partially symmetric tensor}

Suppose that $\mathscr{A}=\left(a_{i j k l}\right)$ is a fourth-order real partially symmetric tensor, where

$$
a_{i j k l}=a_{k j i l}=a_{i l k j}, \quad i, k=1, \ldots, m, j, l=1, \ldots, n .
$$

Here, the dimension of the first and the third indices is $m$, while the dimension of the second and the fourth indices is $n$. Such a fourth-order partially symmetric tensor is useful in quantum physics, see Refs. [12,27].

If $\lambda \in \mathbb{R}, x \in \mathbb{R}^{m}$ and $y \in \mathbb{R}^{n}$ satisfy (6), we call $\lambda$ an M-eigenvalue of $\mathscr{A}$, and call $x$ and $y$ left and right M-eigenvectors of $\mathscr{A}$, associated with the M-eigenvalue $\lambda$.

Let

$$
f(x, y) \equiv \mathscr{A} x y x y \equiv \sum_{i, k=1}^{m} \sum_{j, l=1}^{n} a_{i j k l} x_{i} y_{j} x_{k} y_{l} .
$$

If $f(x, y)>0$ for all $x \in \mathbb{R}^{m}, x \neq 0, y \in \mathbb{R}^{n}, y \neq 0$, then we say that $\mathscr{A}$ is positive definite.

Theorem 1 M-eigenvalues always exist. If $x$ and $y$ are left and right $M$ eigenvectors of $\mathscr{A}$, associated with an $M$-eigenvalue $\lambda$, then

$$
\lambda=\mathscr{A} x y x y .
$$

Proof The feasible region of

$$
\begin{array}{ll}
\min & f(x, y) \equiv \mathscr{A} x y x y \equiv \sum_{i, k=1}^{m} \sum_{j, l=1}^{n} a_{i j k l} x_{i} y_{j} x_{k} y_{l} \\
\text { s.t. } & x^{\mathrm{T}} x=1, \quad y^{\mathrm{T}} y=1
\end{array}
$$

is compact. The objective function of (8) is continuous. Hence, the optimization problem (8) has at least a maximizer and a minimizer. They are critical points of (8) and satisfy (6) with corresponding Lagrangian multipliers. Hence, M-eigenvalues always exist. By (6), we have (7). Clearly, 
$\mathscr{A}$ is positive definite if and only if the optimal value of $(8)$ is positive. Then the last conclusion of the theorem follows.

We may use the last two equations of (6) to homogenize the first two equations of (6). Then we have

$$
\left\{\begin{array}{l}
\mathscr{A} \cdot y x y=\lambda\left(y^{\mathrm{T}} y\right) x, \\
\mathscr{A} x y x \cdot=\lambda\left(x^{\mathrm{T}} x\right) y .
\end{array}\right.
$$

According to algebraic geometry [5], the resultant of (9) is a one-dimensional polynomial $\phi$ of $\lambda$. We call $\phi(\lambda)$ the M-characteristic polynomial of $\mathscr{A}$.

Theorem 2 An M-eigenvalue of $\mathscr{A}$ is always a real root of the $M$ characteristic polynomial $\phi(\lambda)$.

Proof According to the resultant theory [5], (9) has a nonzero complex solution $(x, y)$ if and only if $\lambda$ is a root of the resultant. The conclusion follows.

Define the Frobenius norm of $\mathscr{A}$ as

$$
\|\mathscr{A}\|_{F}=\left(\sum_{i, k=1}^{m} \sum_{j, l=1}^{n}\left(a_{i j k l}\right)^{2}\right)^{1 / 2} .
$$

For $\lambda \in \mathbb{R}, x \in \mathbb{R}^{m}$ and $y \in \mathbb{R}^{n}, \lambda x y x y$ is a rank-one fourth-order partially symmetric tensor with elements $\lambda x_{i} y_{j} x_{k} y_{l}$. We say that $\lambda x y x y$ is the best rank-one approximation of $\mathscr{A}$, if $\lambda \in \mathbb{R}, x \in \mathbb{R}^{m}, x^{\mathrm{T}} x=1$ and $y \in \mathbb{R}^{n}, y^{\mathrm{T}} y=1$ minimize $\|\mathscr{A}-\lambda x y x y\|_{F}$. The best rank-one approximation has wide applications in signal and image processing, wireless communication systems, and independent component analysis, etc. [1,6,9,20,28].

Theorem 3 If $\lambda$ is the $M$-eigenvalue of $\mathscr{A}$ with the largest absolute value, $x$ and $y$ are corresponding left and right $M$-eigenvectors, then $\lambda x y x y$ is the best rank-one approximation of $\mathscr{A}$.

Proof Let $x^{\mathrm{T}} x=1$ and $y^{\mathrm{T}} y=1$. We have

$$
\begin{aligned}
\|\mathscr{A}-\lambda x y x y\|_{F}^{2} & =\|A\|_{F}^{2}-2 \lambda \mathscr{A} x y x y+\lambda^{2}\left(x^{\mathrm{T}} x\right)\left(y^{\mathrm{T}} y\right) \\
& =\|A\|_{F}^{2}-2 \lambda \mathscr{A} x y x y+\lambda^{2} .
\end{aligned}
$$

Its minimum is attained when $\lambda=\mathscr{A} x y x y$. Hence

$$
\begin{aligned}
\min & \left\{\|\mathscr{A}-\lambda x y x y\|_{F}^{2}: \quad \lambda \in \mathbb{R}, x^{\mathrm{T}} x=1, y^{\mathrm{T}} y=1\right\} \\
& =\min \left\{\|\mathscr{A}\|_{F}^{2}-(\mathscr{A} x y x y)^{2}: x^{\mathrm{T}} x=1, y^{\mathrm{T}} y=1\right\} \\
& =\|\mathscr{A}\|_{F}^{2}-\max \left\{(\mathscr{A} x y x y)^{2}: x^{\mathrm{T}} x=1, y^{\mathrm{T}} y=1\right\} .
\end{aligned}
$$

The conclusion follows. 
Suppose that $P=\left(p_{i i^{\prime}}\right) \in \mathbb{R}^{m \times m}$ and $Q=\left(q_{j j^{\prime}}\right) \in \mathbb{R}^{n \times n}$ are orthogonal matrices. Let

$$
b_{i j k l}=\sum_{i^{\prime}, k^{\prime}=1}^{m} \sum_{j^{\prime}, l^{\prime}=1}^{n} p_{i i^{\prime}} q_{j j^{\prime}} p_{k k^{\prime}} q_{l l^{\prime}} a_{i^{\prime} j^{\prime} k^{\prime} l^{\prime}} .
$$

Then $\mathscr{B}=\left(b_{i j k l}\right)$ is also a fourth-order partially symmetric tensor. We say that $\mathscr{A}$ and $\mathscr{B}$ are orthogonally similar.

Theorem 4 If fourth-order partially symmetric tensors $\mathscr{A}$ and $\mathscr{B}$ are orthogonally similar, then they have the same M-eigenvalues. In particular, if they are orthogonally similar via orthogonal matrices $P$ and $Q$ as above, and $\lambda$ is an $M$-eigenvalue of $\mathscr{A}$ with left and right $M$-eigenvectors $x$ and $y$, then $\lambda$ is also an $M$-eigenvalue of $\mathscr{B}$ with left and right M-eigenvectors Px and $Q y$.

The proof of this theorem is similar to the proof of Theorem 7 of Ref. [16]. We omit its proof here.

\section{Strong ellipticity and rank-one positive definiteness}

In this section, we assume that $m=n$.

As the strong ellipticity is related with the M-eigenvalues of $\mathscr{A}$, the rankone positive definiteness of $\mathscr{A}$ is related with the Z-eigenvalues of $\mathscr{A}$. We now review the definition of Z-eigenvalues of $\mathscr{A}$. Clearly, the elasticity tensor $\mathscr{A}$ is rank-one positive definite if the optimal value of the following global polynomial optimization problem is positive:

$$
\begin{array}{ll}
\min & f(x, x) \equiv \mathscr{A} x^{4} \equiv \mathscr{A} x x x x \equiv \sum_{i, j, k, l=1}^{n} a_{i j k l} x_{i} x_{j} x_{k} x_{l} \\
\text { s.t. } & x^{\mathrm{T}} x=1
\end{array}
$$

where $x \in \mathbb{R}^{n}$. Denote $\mathscr{A} x^{3}$ as a vector whose $i$ th component is

$$
\sum_{j, k, l=1}^{n} a_{i j k l} x_{j} x_{k} x_{l} .
$$

The optimality condition of (10) is

$$
\left\{\begin{array}{l}
\mathscr{A} x^{3}=\lambda x, \\
x^{\mathrm{T}} x=1 .
\end{array}\right.
$$

If $\lambda \in \mathbb{R}$ and $x \in \mathbb{R}^{n}$ satisfy (11), we call $\lambda$ a Z-eigenvalue of $\mathscr{A}$, and call $x$ a Z-eigenvector of $\mathscr{A}$, associated with the Z-eigenvalue $\lambda$. Thus, Z-eigenvalues always exist, and $\mathscr{A}$ is rank-one positive definite if and only if its smallest Z-eigenvalue is positive. 
It is easy to see that a Z-eigenvalue is an M-eigenvalue. However, an M-eigenvalue is not necessarily a Z-eigenvalue. We may see this from the following example for $n=2$.

For $n=2$, we have

$$
\begin{aligned}
f(x, y)= & \mathscr{A} x y x y \\
= & a_{1111} x_{1}^{2} y_{1}^{2}+2 a_{1112} x_{1}^{2} y_{1} y_{2}+a_{1212} x_{1}^{2} y_{2}^{2} \\
& +2 a_{1121} x_{1} x_{2} y_{1}^{2}+4 a_{1122} x_{1} x_{2} y_{1} y_{2}+2 a_{1222} x_{1} x_{2} y_{2}^{2} \\
& +a_{2121} x_{2}^{2} y_{1}^{2}+2 a_{2122} x_{2}^{2} y_{1} y_{2}+a_{2222} x_{2}^{2} y_{2}^{2} .
\end{aligned}
$$

Consider the example where

$$
\begin{gathered}
a_{1111}=1, \quad a_{1112}=2, \quad a_{1121}=2, \quad a_{1122}=4, \quad a_{1212}=3, \\
a_{2121}=3, \quad a_{1222}=5, \quad a_{2122}=5, \quad a_{2222}=6 .
\end{gathered}
$$

Then (12) becomes

$$
\begin{aligned}
f(x, y)= & \mathscr{A} x y x y \\
= & x_{1}^{2} y_{1}^{2}+4 x_{1}^{2} y_{1} y_{2}+3 x_{1}^{2} y_{2}^{2}+4 x_{1} x_{2} y_{1}^{2}+16 x_{1} x_{2} y_{1} y_{2} \\
& +10 x_{1} x_{2} y_{2}^{2}+3 x_{2}^{2} y_{1}^{2}+10 x_{2}^{2} y_{1} y_{2}+6 x_{2}^{2} y_{2}^{2} .
\end{aligned}
$$

$\mathscr{A}$ has eight M-eigenvector pairs:

$$
\begin{aligned}
& \left\{\begin{array} { l } 
{ x = ( - 0 . 9 6 3 9 , 0 . 2 6 6 4 ) ^ { \mathrm { T } } , } \\
{ y = ( - 0 . 9 6 3 9 , 0 . 2 6 6 4 ) ^ { \mathrm { T } } , }
\end{array} \quad \left\{\begin{array}{l}
x=(0.5774,0.8165)^{\mathrm{T}}, \\
y=(0.5774,0.8165)^{\mathrm{T}},
\end{array}\right.\right. \\
& \left\{\begin{array} { l } 
{ x = ( - 0 . 8 1 0 1 , 0 . 5 8 6 2 ) ^ { \mathrm { T } } , } \\
{ y = ( - 0 . 8 1 0 1 , 0 . 5 8 6 2 ) ^ { \mathrm { T } } , }
\end{array} \quad \left\{\begin{array}{l}
x=(-0.5774,0.8165)^{\mathrm{T}}, \\
y=(-0.5774,0.8165)^{\mathrm{T}},
\end{array}\right.\right. \\
& \left\{\begin{array} { l } 
{ x = ( 0 . 5 8 9 4 , 0 . 8 0 7 9 ) ^ { \mathrm { T } } , } \\
{ y = ( - 0 . 8 1 6 8 , 0 . 5 7 6 9 ) ^ { \mathrm { T } } , }
\end{array} \quad \left\{\begin{array}{l}
x=(-0.8168,0.5769)^{\mathrm{T}}, \\
y=(0.5894,0.8079)^{\mathrm{T}},
\end{array}\right.\right. \\
& \left\{\begin{array} { l } 
{ x = ( - 0 . 9 3 2 6 , 0 . 3 6 0 9 ) ^ { \mathrm { T } } , } \\
{ y = ( - 0 . 6 4 0 2 , 0 . 7 6 8 2 ) ^ { \mathrm { T } } , }
\end{array} \quad \left\{\begin{array}{l}
x=(-0.6402,0.7682)^{\mathrm{T}}, \\
y=(-0.9326,0.3609)^{\mathrm{T}},
\end{array}\right.\right.
\end{aligned}
$$

and the corresponding M-eigenvalues are, respectively,

$$
\begin{array}{cccc}
0.0710, & 15.2091, & 0.3437, & 0.1242, \\
-1.2765, & -1.2765, & 0.2765, & 0.2765 .
\end{array}
$$

We see that the first four M-eigenvalues are Z-eigenvalues, but the last four M-eigenvalues are not Z-eigenvalues. Thus, the smallest M-eigenvalue is $\lambda=$ -1.2765 , taken at

$$
x=(-0.8168,0.5769)^{\mathrm{T}}, \quad y=(0.5894,0.8079)^{\mathrm{T}}
$$


and

$$
x=(0.5894,0.8079)^{\mathrm{T}}, \quad y=(-0.8168,0.5769)^{\mathrm{T}} .
$$

The strong ellipticity condition does not hold. But the smallest Z-eigenvalue is $0.1242 . \mathscr{A}$ is rank-one positive definite.

We may substitute these M-eigenvalues and M-eigenvectors to (6) to verify them. We may also solve (11) exactly to find the Z-eigenvalues. In fact, (11) is now

$$
\left\{\begin{array}{l}
2 x_{1}^{3}+12 x_{1}^{2} x_{2}+22 x_{1} x_{2}^{2}+10 x_{2}^{3}=\lambda x_{1}, \\
4 x_{1}^{3}+22 x_{1}^{2} x_{2}+30 x_{1} x_{2}^{2}+12 x_{2}^{3}=\lambda x_{2}, \\
x_{1}^{2}+x_{2}^{2}=1
\end{array}\right.
$$

From the second and the third equations, it is easy to see that $x_{2} \neq 0$. Thus, eliminating $\lambda$ from the first two equations and using the third equation, we have

$$
2 t^{4}+10 t^{3}+9 t^{2}-5 t-5=\left(2 t^{2}-1\right)\left(t^{2}+5 t+5\right)=0,
$$

where $t=x_{1} / x_{2}$. This polynomial equation has four roots:

$$
\frac{\sqrt{2}}{2}, \quad-\frac{\sqrt{2}}{2}, \quad \frac{-5+\sqrt{5}}{2}, \quad-\frac{-5+\sqrt{5}}{2} .
$$

For any $t$, the $Z$-eigenvector of $\mathscr{A}$ can be obtained via the relation

$$
x=\frac{1}{\left(1+t^{2}\right)^{1 / 2}}\left(\begin{array}{l}
t \\
1
\end{array}\right) .
$$

Then, the corresponding Z-eigenvalues can be calculated by

$$
\lambda=\mathscr{A} x^{4}=\frac{t^{4}+8 t^{3}+22 t^{2}+20 t+6}{\left(1+t^{2}\right)^{2}} .
$$

We may easily check that the four Z-eigenvalues of $\mathscr{A}$ given earlier are right and there are no other Z-eigenvalues.

Summarizing the above results, we have the following theorem.

Theorem 5 The strong ellipticity condition holds if and only if the smallest $M$-eigenvalue of the elasticity tensor $\mathscr{A}$ is positive. The elasticity tensor $\mathscr{A}$ is rank-one positive definite if and only if its smallest Z-eigenvalue is positive. All the Z-eigenvalues are M-eigenvalues, but not vice versa.

In some cases, it is possible that all the M-eigenvalues are Z-eigenvalues. If, furthermore, for all $i, j, k, l$, we have $a_{i j k l}=a_{j i k l}$, then $\mathscr{A}$ is fully symmetric. In this case, we may denote

$$
\mathscr{A} x y \equiv \mathscr{A} \cdots x y=\left(\sum_{k, l=1}^{n} a_{i j k l} x_{k} y_{l}\right),
$$

which is a symmetric matrix. We have the following theorem. 
Theorem 6 Suppose that $n=2$ and $\mathscr{A}$ is fully symmetric. Then all the $M$-eigenvalues of $\mathscr{A}$ are $Z$-eigenvalues if there are no $x, y \in \mathbb{R}^{2}$, satisfying the following three conditions:

(i) $x^{\mathrm{T}} x=1, y^{\mathrm{T}} y=1$;

(ii) $x$ and $y$ are linearly independent;

(iii) $(\mathscr{A} x y)^{2}=\lambda^{2} I$, where $I$ is the $2 \times 2$ unit matrix.

Proof Suppose that $\lambda$ is an M-eigenvalue with $x$ and $y$ as its left and right Meigenvectors. Then $x^{\mathrm{T}} x=1, y^{\mathrm{T}} y=1$. Suppose that $\lambda$ is not a Z-eigenvalue. Then we may assume that $x$ and $y$ are linearly independent. From (6), we have

$$
\left\{\begin{array}{l}
(\mathscr{A} x y) y=\lambda x \\
(\mathscr{A} x y) x=\lambda y \\
x^{\mathrm{T}} x=1 \\
y^{\mathrm{T}} y=1
\end{array}\right.
$$

Then we have

$$
\left\{\begin{array}{l}
(\mathscr{A} x y)^{2} x=\lambda^{2} x, \\
x^{\mathrm{T}} x=1
\end{array}\right.
$$

and

$$
\left\{\begin{array}{l}
(\mathscr{A} x y)^{2} y=\lambda^{2} y \\
y^{\mathrm{T}} y=1
\end{array}\right.
$$

If $(\mathscr{A} x y)^{2} \neq \lambda^{2} I$, then $x=y$ or $x=-y$, contradicting the assumption that $x$ and $y$ are linearly independent. Hence, $(\mathscr{A} x y)^{2}=\lambda^{2} I$. The conclusion follows now.

Theorem 6 implies that when $\mathscr{A}$ is fully symmetric and $n=2$, it is very possible that all the M-eigenvalues are Z-eigenvalues. It is also possible to extend this theorem to the case that $n=3$. However, it is hard to verify the conditions of this theorem. Hence it is not so useful in practice.

\section{Strong ellipticity and second-order positive definiteness}

The second-order positive definiteness of the elasticity tensor $\mathscr{A}$ has been considered by Lord Kelvin [24,25] 150 years ago , also see Refs. [4,13]. We say that a fourth-order partially symmetric tensor $\mathscr{A}$ is second-order positive definite if for any matrix $D=\left(d_{i j}\right) \in \mathbb{R}^{m \times n}, D \neq 0$, we have

$$
\mathscr{A} D^{2}=\sum_{i, k=1}^{m} \sum_{j, l=1}^{n} a_{i j k l} d_{i j} d_{k l}>0 \text {. }
$$

Actually, in this case, we may regard $D=\left(d_{i j}\right)$ an $m n$-dimensional vector $d$ and $\mathscr{A}$ an $m n \times m n$ symmetric matrix $A$. Then $\mathscr{A}$ is second-order positive 
definite if and only if the smallest eigenvalue $\mu_{\text {min }}$ of this $m n \times m n$ symmetric matrix $A$ is positive. As we discussed in the introduction, when $m=n=3$, the strong ellipticity condition holds if $\mathscr{A}$ is second-order positive definite. However, if the strong ellipticity condition holds, $\mathscr{A}$ may not be second-order positive definite. The following example shows this.

Let $n=2$ and

$$
\begin{gathered}
a_{1111}=1, \quad a_{1112}=2, \quad a_{1122}=4, \quad a_{1212}=12, \quad a_{2121}=12, \\
a_{1222}=1, \quad a_{1121}=2, \quad a_{2122}=1, \quad a_{2222}=2 .
\end{gathered}
$$

Then (12) becomes

$$
\begin{aligned}
f(x, y)= & \mathscr{A} x y x y \\
= & x_{1}^{2} y_{1}^{2}+4 x_{1}^{2} y_{1} y_{2}+12 x_{1}^{2} y_{2}^{2}+4 x_{1} x_{2} y_{1}^{2}+16 x_{1} x_{2} y_{1} y_{2} \\
& +2 x_{1} x_{2} y_{2}^{2}+12 x_{2}^{2} y_{1}^{2}+2 x_{2}^{2} y_{1} y_{2}+2 x_{2}^{2} y_{2}^{2} .
\end{aligned}
$$

$\mathscr{A}$ has six M-eigenvector pairs:

$$
\begin{aligned}
& \left\{\begin{array} { l } 
{ x = ( - 0 . 9 9 4 6 , 0 . 1 0 4 0 ) ^ { \mathrm { T } } , } \\
{ y = ( - 0 . 9 9 4 6 , 0 . 1 0 4 0 ) ^ { \mathrm { T } } , }
\end{array} \quad \left\{\begin{array}{l}
x=(-0.6723,0.7403)^{\mathrm{T}}, \\
y=(-0.6723,0.7403)^{\mathrm{T}},
\end{array}\right.\right. \\
& \left\{\begin{array} { l } 
{ x = ( 0 . 6 3 0 9 , 0 . 7 7 5 9 ) ^ { \mathrm { T } } , } \\
{ y = ( - 0 . 6 1 6 7 , 0 . 7 8 7 2 ) ^ { \mathrm { T } } , }
\end{array} \quad \left\{\begin{array}{l}
x=(0.7153,0.6989)^{\mathrm{T}}, \\
y=(0.7153,0.6989)^{\mathrm{T}},
\end{array}\right.\right. \\
& \left\{\begin{array} { l } 
{ x = ( - 0 . 6 1 6 7 , 0 . 7 8 7 2 ) ^ { \mathrm { T } } , } \\
{ y = ( 0 . 6 3 0 9 , 0 . 7 7 5 9 ) ^ { \mathrm { T } } , }
\end{array} \quad \left\{\begin{array}{l}
x=(-0.0562,0.9984)^{\mathrm{T}}, \\
y=(-0.0562,0.9984)^{\mathrm{T}},
\end{array}\right.\right.
\end{aligned}
$$

and the corresponding M-eigenvalues are, respectively,

$$
0.5837, \quad 7.8222, \quad 2.7964, \quad 13.7558, \quad 2.7964, \quad 1.8882 .
$$

Since the smallest $M$-eigenvalue is $0.5837>0$, we see that the strong ellipticity condition holds.

The corresponding $4 \times 4$ matrix of $\mathscr{A}$ is

$$
\left(\begin{array}{cccc}
a_{1111} & a_{1112} & a_{1121} & a_{1122} \\
a_{1112} & a_{1212} & a_{1122} & a_{1222} \\
a_{1121} & a_{1222} & a_{2121} & a_{2122} \\
a_{1122} & a_{1122} & a_{2122} & a_{2222}
\end{array}\right)=\left(\begin{array}{cccc}
1 & 2 & 2 & 4 \\
2 & 12 & 4 & 1 \\
2 & 4 & 12 & 1 \\
4 & 1 & 1 & 2
\end{array}\right),
$$

whose four eigenvalues are

$$
-2.6110, \quad 4.7779, \quad 8.0000, \quad 16.8331 \text {. }
$$

Since the smallest eigenvalue $-2.6110<0$, we can see that $\mathscr{A}$ is not secondorder positive definite. 


\section{A direct method for M-eigenvalues and M-eigenvectors when $m=n=2$}

In this section, we present a direct method to find all the $M$-eigenvalues and $M$-eigenvector pairs. The key idea here is to reduce the five variables system (6) to a system involving only two variables. Then, for this system of two variables, we may use the Sylvester formula of the resultant to find the solutions.

We have the following theorem.

Theorem 7 We have the following results on the $M$-eigenvalues and their corresponding $M$-eigenvector pairs.

(a) If $a_{1112}=a_{1121}=0$, then $\lambda=a_{1111}$ is an $M$-eigenvalue of $\mathscr{A}$ and the corresponding $M$-eigenvector pair is $x=y=(1,0)^{\mathrm{T}}$.

(b) For any real roots $(u, v)^{\mathrm{T}}$ of the following equations:

$$
\begin{aligned}
& \left\{\begin{array}{l}
a_{1121} u^{2}+\left(a_{2121}-a_{1111}\right) u v-a_{1121} v^{2}=0, \\
a_{1112} u^{2}+2 a_{1122} u v+a_{2122} v^{2}=0
\end{array}\right. \\
& \lambda=a_{1111} u^{2}+2 a_{1121} u v+a_{2121} v^{2}
\end{aligned}
$$

is an $M$-eigenvalue with the corresponding eigenvector pair

$$
x=\frac{(u, v)^{\mathrm{T}}}{\sqrt{u^{2}+v^{2}}}, \quad y=( \pm 1,0)^{\mathrm{T}} .
$$

(c) For any real roots $(u, v)^{\mathrm{T}}$ of the following equations:

$$
\left\{\begin{array}{c}
a_{1121} u^{2}+2 a_{1122} u v+a_{1222} v^{2}=0 \\
a_{1112} u^{2}+\left(a_{1212}-a_{1111}\right) u v-a_{1112} v^{2}=0 \\
\lambda=a_{1111} u^{2}+2 a_{1112} u v+a_{1212} v^{2}
\end{array}\right.
$$

is an $M$-eigenvalue with the corresponding eigenvector pair

$$
x=( \pm 1,0)^{\mathrm{T}}, \quad y=\frac{(u, v)^{\mathrm{T}}}{\sqrt{u^{2}+v^{2}}} .
$$

(d) $\lambda=\mathscr{A} x y x y$ is an $M$-eigenvalue and

$$
x= \pm \frac{1}{\sqrt{u^{2}+1}}(u, 1)^{\mathrm{T}}, \quad y= \pm \frac{1}{\sqrt{v^{2}+1}}(v, 1)^{\mathrm{T}}
$$

constitute an $M$-eigenvector pair, where $u$ and $v$ are real solutions of the following system of polynomial equations:

$$
\left\{\begin{array}{c}
a_{1121} u^{2} v^{2}+2 a_{1122} u^{2} v+a_{1222} u^{2}+\left(a_{2121}-a_{1111}\right) u v^{2}-a_{1121} v^{2} \\
+2\left(a_{2122}-a_{1112}\right) u v+\left(a_{2222}-a_{1212}\right) u-2 a_{1122} v-a_{1222}=0 \\
a_{1112} u^{2} v^{2}+\left(a_{1212}-a_{1111}\right) u^{2} v-a_{1112} u^{2}+2\left(a_{1222}-a_{1121}\right) u v \\
+2 a_{1122} u v^{2}-2 a_{1122} u+a_{2122} v^{2}+\left(a_{2222}-a_{2121}\right) v-a_{2122}=0
\end{array}\right.
$$


All the $M$-eigenvalues and the associated $M$-eigenvector pairs are given by (a)-(d) if $a_{1112}=a_{1121}=0$, and by (b)-(d) otherwise.

Proof (a) If $a_{1112}=a_{1121}=0$, it is direct to check that (a) holds.

(b) If $y_{2}=0$, then $y_{1}= \pm 1$ and (6) becomes

$$
\left\{\begin{array}{l}
a_{1111} x_{1}+a_{1121} x_{2}=\lambda x_{1} \\
a_{1121} x_{1}+a_{2121} x_{2}=\lambda x_{2} \\
a_{1111} x_{1}^{2}+2 a_{1121} x_{1} x_{2}+a_{2121} x_{2}^{2}=\lambda \\
a_{1112} x_{1}^{2}+2 a_{1122} x_{1} x_{2}+a_{2122} x_{2}^{2}=0 \\
x_{1}^{2}+x_{2}^{2}=1 .
\end{array}\right.
$$

Eliminating $\lambda$ from the first two equations, we get

$$
\left\{\begin{array}{l}
a_{1121} x_{1}^{2}+\left(a_{2121}-a_{1111}\right) x_{1} x_{2}-a_{1121} x_{2}^{2}=0 \\
a_{1112} x_{1}^{2}+2 a_{1122} x_{1} x_{2}+a_{2122} x_{2}^{2}=0 \\
x_{1}^{2}+x_{2}^{2}=1
\end{array}\right.
$$

Let

$$
u=\frac{x_{1}}{\sqrt{x_{1}^{2}+x_{2}^{2}}}, \quad v=\frac{x_{2}}{\sqrt{x_{1}^{2}+x_{2}^{2}}} .
$$

Then the results of (b) follows immediately.

(c) It can be proved in a similar way as (b).

(d) If $x_{2} \neq 0$ and $y_{2} \neq 0$, (6) becomes

$$
\left\{\begin{array}{l}
a_{1111} x_{1} y_{1}^{2}+2 a_{1112} x_{1} y_{1} y_{2}+a_{1212} x_{1} y_{2}^{2}+a_{1121} x_{2} y_{1}^{2} \\
\quad+2 a_{1122} x_{2} y_{1} y_{2}+a_{1222} x_{2} y_{2}^{2}=\lambda x_{1}, \\
a_{1121} x_{1} y_{1}^{2}+2 a_{1122} x_{1} y_{1} y_{2}+a_{1222} x_{1} y_{2}^{2}+a_{2121} x_{2} y_{1}^{2} \\
\quad+2 a_{2122} x_{2} y_{1} y_{2}+a_{2222} x_{2} y_{2}^{2}=\lambda x_{2}, \\
a_{1111} x_{1}^{2} y_{1}+a_{1112} x_{1}^{2} y_{2}+2 a_{1121} x_{1} x_{2} y_{1}+2 a_{1122} x_{1} x_{2} y_{2} \\
\quad+a_{2121} x_{2}^{2} y_{1}+a_{2122} x_{2}^{2} y_{2}=\lambda y_{1}, \\
a_{1112} x_{1}^{2} y_{1}+a_{1212} x_{1}^{2} y_{2}+2 a_{1122} x_{1} x_{2} y_{1}+2 a_{1222} x_{1} x_{2} y_{2} \\
\quad+a_{2122} x_{2}^{2} y_{1}+a_{2222} x_{2}^{2} y_{2}=\lambda y_{2}, \\
x_{1}^{2}+x_{2}^{2}=1, \\
y_{1}^{2}+y_{2}^{2}=1 .
\end{array}\right.
$$

Let $u=x_{1} / x_{2}$ and $v=y_{1} / y_{2}$. Then from the first two equalities of the above system, we have

$$
\begin{aligned}
& a_{1121} u^{2} v^{2}+2 a_{1122} u^{2} v+a_{1222} u^{2}+\left(a_{2121}-a_{1111}\right) u v^{2}-a_{1121} v^{2} \\
& +2\left(a_{2122}-a_{1112}\right) u v+\left(a_{2222}-a_{1212}\right) u-2 a_{1122} v-a_{1222}=0
\end{aligned}
$$


and from the third and the fourth equalities, we have

$$
\begin{aligned}
& a_{1112} u^{2} v^{2}+\left(a_{1212}-a_{1111}\right) u^{2} v-a_{1112} u^{2}+2\left(a_{1222}-a_{1121}\right) u v \\
& \quad+2 a_{1122} u v^{2}-2 a_{1122} u+a_{2122} v^{2}+\left(a_{2222}-a_{2121}\right) v-a_{2122}=0 .
\end{aligned}
$$

Combining the above two equalities and the assumption that $x_{1}^{2}+x_{2}^{2}=1$ and $y_{1}^{2}+y_{2}^{2}=1$, we get the assertion immediately.

From Theorem 7 , we can see that to find all the $M$-eigenvalues and the associated $M$-eigenvector pairs, we need to solve some systems of polynomial equations with two variables. To solve such systems, we can use the resultant method from algebraic geometry. For example, to solve (19), we may regard it as equations of $u$,

$$
\left\{\begin{array}{c}
\alpha_{0} u^{2}+\alpha_{1} u+\alpha_{2}=0 \\
\beta_{0} u^{2}+\beta_{1} u+\beta_{2}=0
\end{array}\right.
$$

where

$$
\begin{gathered}
\alpha_{0}=a_{1121} v^{2}+2 a_{1122} v+a_{1222}, \\
\alpha_{1}=\left(a_{2121}-a_{1111}\right) v^{2}+2\left(a_{2122}-a_{1112}\right) v+\left(a_{2222}-a_{1212}\right), \\
\alpha_{2}=-\left(a_{1121} v^{2}+2 a_{1122} v+a_{1222}\right), \\
\beta_{0}=a_{1112} v^{2}+a_{1212} v-a_{1112}, \\
\beta_{1}=2 a_{1122} v^{2}+2\left(a_{1222}-a_{1121}\right) v-2 a_{1122}, \\
\left.\beta_{2}=a_{2122} v^{2}+\left(a_{2222}-a_{2121}\right) v-a_{2122}\right) .
\end{gathered}
$$

System (20) has solutions if and only if its resultant vanishes [5]. By the Sylvester theorem [5], its resultant can be calculated as a determinant of the following $4 \times 4$ matrix:

$$
\left(\begin{array}{cccc}
\alpha_{0} & \alpha_{1} & \alpha_{2} & 0 \\
0 & \alpha_{0} & \alpha_{1} & \alpha_{2} \\
\beta_{0} & \beta_{1} & \beta_{2} & 0 \\
0 & \beta_{0} & \beta_{1} & \beta_{2}
\end{array}\right),
$$

which is a polynomial of $v$. We can use Matlab to find all of its real roots. After this, we substitute them to (19) to find all the real solutions of $u$. Correspondingly, all the $M$-eigenvalues and the associated $M$-eigenvector pairs can be found.

\section{SOS method for smallest M-eigenvalue and its M-eigenvectors when $m=n=3$}

When $m=n=3$, it is not easy to get all the $M$-eigenvalues and the associated $M$-eigenvector pairs. In some cases, we just want to know if the 
strong ellipticity holds or not, which is equivalent to find if the objective value of (4) is positive or not.

A sufficient condition to ensure the objective value of (4) is positive is that there is some positive number $\varepsilon$, such that for all $x, y$,

$$
x_{1}^{2}+x_{2}^{2}=1, \quad y_{1}^{2}+y_{2}^{2}=1,
$$

and

$$
f(x, y)-\varepsilon=\mathscr{A} x y x y-\varepsilon
$$

is the sum of squares of some polynomials, i.e., there are some polynomials $g_{1}(x, y), g_{2}(x, y)$, and $s_{1}(x, y), s_{2}(x, y), \ldots, s_{m}(x, y)$,

$$
f(x, y)-\varepsilon=g_{1}(x, y)\left(x_{1}^{2}+x_{2}^{2}-1\right)+g_{2}(x, y)\left(y_{1}^{2}+y_{2}^{2}-1\right)+\sum_{i=1}^{t} s_{i}(x, y)^{2} .
$$

Verifying the above condition is also a difficult task, since the degrees of the polynomials $g_{1}(x, y), g_{2}(x, y)$ and $s_{i}(x, y), i=1, \ldots, m$, are unknown. If we further restrict the degree of the polynomials $g_{1}(x, y), g_{2}(x, y)$ and $s_{i}(x, y), i=1, \ldots, t$, to be no larger than a positive number $d$, then $(23)$ is equivalent to a semidefinite programming problem, which can be solved via interior point algorithms efficiently. For details of the SOS method solving optimization problems with polynomials, the readers are referred to Refs. $[10,15]$.

\section{$7 \quad$ Final remarks}

In this paper, we studied the strong ellipticity condition via M-eigenvalues. The strong ellipticity condition holds if and only if the smallest M-eigenvalue of the elasticity tensor is positive. A Z-eigenvalue of the elasticity tensor is an M-eigenvalue but not vice versa. In fact, it is relatively easier to find all the Z-eigenvalues and the associated Z-eigenvectors than to find all the Meigenvalues and the associated M-eigenvectors. In Section 5, we presented a direct method to find all the M-eigenvalues and the associated M-eigenvectors in the case $m=n=2$. When $m=n=3$, this task becomes difficult. However, we may use a direct method to find all the Z-eigenvalues and the associated Z-eigenvectors in the case $m=n=3$. Note that if an M-eigenvalue is not a Z-eigenvalue, then it appears twice, as if we switch the left and the right M-eigenvectors, we get the same M-eigenvalue. Can we use this property to derive a direct method to find all the M-eigenvalues which are not Z-eigenvalues, when $m=n=3$ ? We shall leave this for future investigation.

Problem (9) has applications in quantum physics and was proved to be NP-hard in Ref. [12]. It is called a bi-quadratical programming problem there. A practical method to solve it was presented in Ref. [27].

Recently, Chang, Pearson and Zhang [3] have unified the definitions of $\mathrm{H}-$ eigenvalues, Z-eigenvalues and D-eigenvalues in Refs. [11,16,19,21]. What 
is its relation with M-eigenvalues here? Chang, Pearson and Zhang [2] established the Perron-Frobenius theorem for nonnegative tensors. If tensor $\mathscr{A}$ is nonnegative, will problem (4) have some better solution methods?

Acknowledgements The authors are thankful to the referees for their comments, which improved the paper. This work was supported by the Research Grant Council of Hong Kong and a Hong Kong Polytechnic University Chair Professor Fund.

\section{References}

1. Cardoso J F. High-order contrasts for independent component analysis. Neural Computation, 1999, 11: 157-192

2. Chang K C, Pearson K, Zhang T. Perron-Frobenius theorem for nonnegative tensors. Commu Math Sci, 2008, 6: 507-520

3. Chang K C, Pearson K, Zhang T. On eigenvalue problems of real symmetric tensors. Journal of Mathematical Analysis and Applications, 2009, 350: 416-422

4. Basser P J, Pajevic S. Spectral decomposition of a 4th-order covariance tensor: Applications to diffusion tensor MRI. Signal Processing, 2007, 87: 220-236

5. Cox D, Little J, O'Shea D. Using Algebraic Geometry. New York: Springer-Verlag, 1998

6. De Lathauwer L, De Moor B, Vandewalle J. On the best rank-1 and rank- $\left(R_{1}, R_{2}\right.$, $\left.\ldots, R_{N}\right)$ approximation of higher-order tensor. SIAM J Matrix Anal Appl, 2000, 21: $1324-1342$

7. Knowles J K, Sternberg E. On the ellipticity of the equations of non-linear elastostatics for a special material. J Elasticity, 1975, 5: 341-361

8. Knowles J K, Sternberg E. On the failure of ellipticity of the equations for finite elastostatic plane strain. Arch Ration Mech Anal, 1977, 63: 321-336

9. Kofidis E, Regalia P A. On the best rank-1 approximation of higher-order supersymmetric tensors. SIAM J Matrix Anal Appl, 2002, 23: 863-884

10. Lasserre J B. Global optimization with polynomials and the problems of moments. SIAM Journal on Optimization, 2001, 11: 796-817

11. Lim L -H. Singular values and eigenvalues of tensors: a variational approach. In: Proceedings of the IEEE International Workshop on Computational Advances in Multi-Sensor Adaptive Processing (CAMSAP '05), Vol 1. 2005, 129-132

12. Ling C, Nie J, Qi L, Ye Y. SDP and SOS relaxations for bi-quadratic optimization over unit spheres. Department of Applied Mathematics, The Hong Kong Polytechnic University, July 2008. Manuscript

13. Morse P M, Feschbach H. Methods of Theoretic Physics, Vol 1. New York: McGrawHill, 1979, 519

14. Ni G, Qi L, Wang F, Wang Y. The degree of the E-characteristic polynomial of an even order tensor. J Math Anal Appl, 2007, 329: 1218-1229

15. Parrilo P A. Semidefinite programming relaxation for semialgebraic Problems. Mathematical Programming, 2003, 96: 293-320

16. Qi L. Eigenvalues of a real supersymmetric tensor. J Symbolic Computation, 2005, 40: $1302-1324$

17. Qi L. Rank and eigenvalues of a supersymmetric tensor, a multivariate homogeneous polynomial and an algebraic surface defined by them. J Symbolic Computation, 2006, 41: 1309-1327

18. Qi L. Eigenvalues and invariants of tensors. J Math Anal Appl, 2007, 325: 13631377

19. Qi L, Sun W, Wang Y. Numerical multilinear algebra and its applications. Frontiers of Mathematics in China, 2007, 2(4): 501-526

20. Qi L, Wang F, Wang Y. Z-eigenvalue methods for a global polynomial optimization problem. Mathematical Programming, 2009, 118: 301-316 
21. Qi L, Wang Y, Wu E X. D-eigenvalues of diffusion kurtosis tensor. Journal of Computational and Applied Mathematics, 2008, 221: 150-157

22. Rosakis P. Ellipticity and deformations with discontinuous deformation gradients in finite elastostatics. Arch Ration Mech Anal, 1990, 109: 1-37

23. Simpson H C, Spector S J. On copositive matrices and strong ellipticity for isotropic elastic materials. Arch Rational Mech Anal, 1983, 84: 55-68

24. Thomson W (Lord Kelvin). Elements of a mathematical theory of elasticity. Philos Trans R Soc, 1856, 166: 481

25. Thomson W (Lord Kelvin). Elasticity. Encyclopedia Briannica, Vol 7. 9th Ed. London, Edingburgh: Adam and Charles Black, 1878, 796-825

26. Wang Y, Aron M. A reformulation of the strong ellipticity conditions for unconstrained hyperelastic media. Journal of Elasticity, 1996, 44: 89-96

27. Wang Y, Qi L, Zhang X. A practical method for computing the largest M-eigenvalue of a fourth-order partially symmetric tensor. Numerical Linear Algebra with Applications (to appear)

28. Zhang T, Golub G H. Rank-1 approximation of higher-order tensors. SIAM J Matrix Anal Appl, 2001, 23: 534-550 